\title{
CLASS NUMBERS OF DEFINITE QUATERNARY FORMS WITH NONSQUARE DISCRIMINANT
}

\author{
BY PAUL PONOMAREV ${ }^{1}$ \\ Communicated by Nathan Jacobson, November 2, 1972
}

1. Introduction. Let $V$ be a definite quadratic space of dimension four over the field of rational numbers $Q$. If the discriminant $\Delta(V)$ is square, then the number of classes of maximal integral lattices can be computed by means of the formulas given by the author in [6] and [7]. The purpose of this note is to announce analogous class number formulas for the case where $\Delta(V)$ is not square, $V_{p}$ is isotropic for each finite prime $p$, and the norm of the fundamental unit of $K=Q\left((\Delta(V))^{1 / 2}\right)$ is -1 .

Let $\mathfrak{M}$ denote the genus of maximal integral lattices of $V, \mathfrak{I}$ the idealcomplex containing $\mathfrak{M}$. Let $\Delta$ denote the discriminant of $\mathfrak{M}$. Then $\mathfrak{I}$ can also be described as the set of all maximal lattices having reduced determinant $\Delta[1, p$. 87]. The formulas we present here are for the number of (proper) similitude classes in $\mathfrak{I}$. We denote this class number by $H$. The number of classes in $\mathfrak{M}$ will be denoted by $H_{0}$. The idealcomplex $\mathfrak{I}$ decomposes into $g^{+}$similitude genera, where $g^{+}=$the number of strict genera of $K$ [5, p. 338]. The similitude genus containing $\mathfrak{M}$ has $H_{0}$ similitude classes. It follows that $H_{0} \leqq H$. Equality holds if and only if $K$ has prime discriminant, since $g^{+}=1$ in that case (cf. Corollary below).

2. Preliminaries. Let $C_{V}^{+}$denote the second Clifford algebra of $V$. Then $C_{V}^{+}=\mathfrak{A}_{K}=\mathfrak{A} \otimes_{\mathbb{Q}} K$, where $\mathfrak{U}$ is a definite quaternion algebra over $\boldsymbol{Q}$. Let $\alpha \mapsto \alpha^{*}$ be the canonical involution of $\mathfrak{U}_{K}$ and $N: \mathfrak{U}_{K} \rightarrow K$ the (reduced) norm mapping. The conjugation $x \mapsto \bar{x}$ of $K$ can be extended to a $Q$ automorphism $\alpha \mapsto \bar{\alpha}$ of $\mathfrak{U}_{K}$ so that $\mathfrak{A}$ is its ring of fixed elements. Let $W$ be the set of all $\alpha$ in $\mathfrak{A}_{K}$ such that $\alpha=\bar{\alpha}^{*}$. Then $W$ is a four-dimensional $Q$ subspace of $\mathfrak{U}_{K}$ and the restriction of $N$ to $W$ takes values in $Q$. In this way $W$ may be regarded as a quadratic space over $\boldsymbol{Q}$. We assume, without loss of generality, that $V$ is positive definite. Then $V$ is isometric to $W$. In particular, the condition that $V_{p}$ is isotropic for every finite prime $p$ is equivalent to the condition that $\mathfrak{A}$ splits at every finite prime which splits in $K$. From this it follows, by a straightforward computation of local discriminants, that

$$
\Delta=\Delta_{K}\left(p_{1} \cdots p_{e}\right)^{2},
$$

where $\Delta_{K}$ is the discriminant of $K$ and $p_{1}, \ldots, p_{e}$ are all the nonsplit finite

AMS (MOS) subject classifications (1970). Primary 10C05, 12A80; Secondary $12 \mathrm{~A} 85$.

${ }^{1}$ Partially supported by NSF grant GP-25320. 
primes of $\mathfrak{A}$ which remain prime in $K$. We put $\delta=p_{1} \cdots p_{e}$.

3. Statement of results. Let $D$ denote the square-free kernel of $\Delta_{K}$. For any positive integer $m$ let $\lambda(m)$ denote the number of primes dividing $m$; let $h(-m)$ denote the class number of the imaginary quadratic extension $Q\left((-m)^{1 / 2}\right)$. Denote the Minkowski-Siegel weight of $\mathfrak{I}$ by $M(\mathfrak{I})$.

THEOREM 1. Suppose that $V$ satisfies the conditions stated in the Introduction. If $D$ is odd and $\Delta>5$, then

$$
\begin{aligned}
H= & M(\mathfrak{I})+c_{1} h(-D)+c_{3} h(-3 D) \\
& +\sum_{n|\delta, d| D} 2^{-\lambda(n)-\sigma(n d)} h(-n d) h(-n D / d),
\end{aligned}
$$

where $n d>3, d<D^{1 / 2}$ and

$$
\begin{aligned}
c_{1}=\frac{1}{8} & \text { if } 2 \nmid \delta, \\
=\frac{3}{16} & \text { if } 2 \mid \delta, \\
c_{3}=\frac{1}{6} & \text { if } 3 \nmid \delta, \\
=\frac{5}{6} & \text { if } 3 \mid \delta, D \equiv 1(\bmod 8), \\
=\frac{1}{3} & \text { if } 3 \mid \delta, D \equiv 5(\bmod 8),
\end{aligned}
$$

and if $D \equiv 1(\bmod 8)$,

$$
\begin{aligned}
\sigma(m) & =-2 & & \text { if } m \equiv 3(\bmod 8) \\
& =0 & & \text { if } m \equiv 7(\bmod 8) \\
& =2 & & \text { if } m \equiv 1(\bmod 4)
\end{aligned}
$$

while if $D \equiv 5(\bmod 8)$,

$$
\begin{aligned}
\sigma(m) & =0 \quad \text { if } m \equiv 3(\bmod 4), \\
& =2 \quad \text { if } m \equiv 2(\bmod 4), \\
& =2 \quad \text { if } m \equiv 1(\bmod 4), 2 \nmid \delta, \\
& =3 \quad \text { if } m \equiv 1(\bmod 4), 2 \mid \delta .
\end{aligned}
$$

Furthermore,

$$
M(\mathfrak{J})=\frac{\prod_{p \mid \delta}\left(p^{2}+1\right)}{\left.3 \cdot 2^{e+2}\left(\frac{D}{2}\right)-4\right)}\left[\sum_{m=1}^{(D-1) / 2}\left(\frac{D}{m}\right) m\right],
$$

where $\left(\frac{D}{m}\right)$ is the Kronecker symbol.

REMARK. Since the fundamental unit of $K$ has norm equal to -1 , every 
prime divisor of $D$ is congruent to $1(\bmod 4)$. Hence $D$ itself must be congruent to $1(\bmod 4)$.

COROLlaRY. Suppose that $\Delta=p$, a prime greater than 5. Then

$$
H_{0}=H=\frac{\sum_{m=1}^{(p-1) / 2}\left(\frac{p}{m}\right) m}{12\left(\left(\frac{p}{2}\right)-4\right)}+\frac{h(-p)}{8}+\frac{h(-3 p)}{6} .
$$

Remarks. 1. If $\Delta=p$, a prime, then (1) implies $\Delta_{K}=p$. In this case it is well known that the norm of the fundamental unit of $K$ is -1 .

2. Tamagawa has shown, under the assumption of the Corollary, that $H=h\left(\mathfrak{A}_{\mathbf{K}}\right) / h(K)$, where $h\left(\mathfrak{A}_{\mathbf{K}}\right)$ is the ideal class number of $\mathfrak{A}_{\mathbf{K}}$ and $h(K)$ is the ideal class number of $K$. Combining this with Peters' formula for $h\left(\mathfrak{A}_{K}\right)[5$, p. 363], we obtain another proof of (4).

3. In the classical terminology, (4) is a formula for the number of classes of integral quaternary forms of discriminant $p$.

THEOREM 2. Suppose that $V$ satisfies the conditions stated in the Introduction. If $D$ is even, then

$$
\begin{aligned}
H= & M(\mathfrak{J})+\frac{5}{8} h(-D)+c_{2} h(-D / 2)+c_{3} h(-3 D) \\
& +\sum_{n|\delta, d| D} c_{n d} 2^{-\lambda(n)-\sigma(n d)} h(-n d) h(-n D / d),
\end{aligned}
$$

where $n d>3, d<D^{1 / 2}$ and

$$
\begin{aligned}
c_{2}=0 & \text { if } D=2, \\
=\frac{3}{4} & \text { if } D \neq 2, \\
c_{3}=\frac{1}{6} & \text { if } 3 \Varangle \delta, \\
=\frac{7}{12} & \text { if } 3 \mid \delta,
\end{aligned}
$$

and for $m>3$,

$$
\begin{aligned}
& c_{m}=5 \text { if } m=3(\bmod 8) \text {, } \\
& =1 \text { if } m=7(\bmod 8) \text {, } \\
& =3 \text { if } m=1(\bmod 4) \text {, } \\
& \sigma(m)=1 \quad \text { if } m=3(\bmod 8) \text {, } \\
& =0 \quad \text { if } m=7(\bmod 8) \text {, } \\
& =2 \text { if } m=1(\bmod 4) \text {. }
\end{aligned}
$$

Furthermore, 


$$
\begin{aligned}
M(\mathfrak{I}) & =\frac{\prod_{p \mid \delta}\left(p^{2}+1\right)}{3 \cdot 2^{e+3}}\left[\sum_{m=1}^{D / 2}\left(\frac{4 D}{m}\right)\left(D+\left((-1)^{(m-1) / 2}-1\right) m\right)\right] \text { if } D \neq 2, \\
& =\prod_{p \mid \delta} \frac{\left(p^{2}+1\right)}{3 \cdot 2^{e+3}} \text { if } D=2 .
\end{aligned}
$$

4. Outline of the proof. If $V$ is regarded as the subspace of all elements in $\mathfrak{A}_{K}$ fixed by $\alpha \mapsto \bar{\alpha}^{*}$, then the proper similitudes of $V$ are all the mappings of the form $\mu \mapsto r \alpha \mu \bar{\alpha}^{*}$, where $r, \alpha$ are invertible elements of $Q, \mathfrak{N}_{K}$, respectively. Using this description of the group of proper similitudes, we deduce that $H=t_{\delta}$, the type number of all orders of level $\delta$ in $\mathfrak{A}_{K}[\mathbf{2}$, p. 130]. Denote the multiplicative groups of $K, \mathfrak{U}_{K}$ by $K^{\times}, \mathfrak{U}_{K}^{\times}$, respectively, and their ideal groups by $J_{K}, J_{\mathfrak{U}_{K}}$, respectively. Put $G=\mathfrak{Q}_{K}^{\times} / K^{\times}, G_{A}$ $=J_{\mathfrak{Q}_{K}} / J_{K}$. Then $G_{A}$ acts transitively on the collection of orders of level $\delta$ by conjugation. Fix an order $\Omega$ of $\mathfrak{U}_{K}$ of level $\delta$ and denote its isotropy group under the action of $G_{\boldsymbol{A}}$ by $G_{\tilde{\Omega}}$. Then we have

$$
t_{\delta}=\operatorname{card}\left(G_{\tilde{\Omega}} \backslash G_{\boldsymbol{A}} / G\right) \text {. }
$$

Proceeding as in [6], we regard $t_{\delta}$ as the trace of the convolution operator $f \mapsto F_{\tilde{\Omega}} * f$ on $L_{2}\left(G_{\tilde{\Omega}} \mid G_{A} / G\right)$, where $F_{\tilde{\Omega}}$ is the characteristic function of $G_{\tilde{\Omega}}$. We fix a representative $s$ from each conjugacy class of $G$ and denote the centralizer of $s$ in $G$ by $G(s)$. Applying the Selberg trace formula, we obtain

$$
H=t_{\delta}=\sum_{s} \int_{G_{\mathrm{A}} / G(s)} \psi_{s}\left(g^{\prime}\right) d g^{\prime},
$$

where $\psi_{s}\left(g^{\prime}\right)=F_{\tilde{\Omega}}\left(g s g^{-1}\right)$ for $g$ in $G_{\boldsymbol{A}}$.

NotATION. If $\alpha$ is an element of $\mathfrak{A}_{K}$ and $x$ is an algebraic number, then $\alpha \sim x$ will mean that $\alpha$ and $x$ have the same minimal polynomial over $K$.

Making essential use of the conditions stated in the Introduction, we can show that a complete set of representatives for the conjugacy classes with nonzero contributions to (7) is given by $\left\{\alpha \bmod K^{\times}\right\}$, where

(i) $\alpha \sim 1$;

(ii) $\alpha \sim \sqrt{-1}, \zeta$, where $\zeta$ is a primitive cube root of unity;

(iii) $\alpha \sim 1+\sqrt{-1}, \sqrt{-2}$ if $2 \mid \Delta$;

(iv) $\alpha \sim \sqrt{-3}, \zeta \sqrt{-3}$ if $3 \mid \Delta$;

(v) $\alpha \sim \sqrt{-m}$ if $m \mid \delta D$.

The contribution of (i) is $M(\mathfrak{I})$, which is evaluated by means of Leopoldt's formula for $L(2, \chi)$, where $\chi(m)=\left(\Delta_{K} / m\right)[4$, p. 135]. The resulting expression is simplified as in $[3, \S 6]$ to yield (3) and (6). The contribution of each of the remaining $\alpha$ in (ii)-(v) is evaluated and found to be a simple rational multiple of the relative class number of $K(\alpha)$ over $K$. The classical formula of Bachmann for the class number of a bicyclic biquadratic imaginary 
extension of $\boldsymbol{Q}$ is then invoked to yield the final formulas.

\section{REFERENCES}

1. M. Eichler, Quadratische Formen und orthogonale Gruppen, Die Grundlehren der math. Wissenschaften in Einzeldarstellungen mit besonderer Berücksichtigung der Anwendungsgebiete, Band 63, Springer-Verlag, Berlin, 1952. MR 14, 540.

2. (1955), 127-151. MR 18, 297.

3. O. Körner, Die Masse der Geschlechter quadratischer Formen vom Range $\leqq 3$ in quadratischen Zahlkörpern, Math. Ann. 193 (1971), 279-314.

4. H.-W. Leopoldt, Eine Verallgemeinerung der Bernoullischen Zahlen, Abh. Math. Sem. Univ. Hamburg 22 (1958), 131-140. MR 19, 1161.

5. M. Peters, Ternäre und quaternäre quadratische Formen und Quaternionenalgebren, Acta Arith. 15 (1968/1969), 329-365. MR 40 \#2605.

6. P. Ponomarev, Class numbers of positive definite quaternary forms, Bull. Amer. Math. Soc. 76 (1970), 646-649. MR 41 \# 3396.

7. - Class numbers of definite quaternary forms with square discriminant, J. Number Theory (to appear).

Department of Mathematics, The Johns Hopkins University, Baltimore, Maryland 21218 